\title{
Mimosa chacoënsis (Leguminosae), NUEVA CitA PARA LA FLORA ARGENTINA
}

\author{
MATÍAS MORALES ${ }^{1,2,3, *}$ y RENÉE H. FORTUNATO ${ }^{1,2,3}$
}

\begin{abstract}
Resumen: Mimosa chacoënsis, perteneciente a la sección Habbasia serie Rojasianae del género Mimosa, es citada por primera vez para la Argentina, en la provincia de Formosa. Esta especie, endémica de la ecorregión del Chaco Seco, previamente había sido registrada sólo para Bolivia y Paraguay. Como resultado de este trabajo se eleva a 56 el número de especies conocidas de Mimosa para la Flora Argentina. Se presenta para esta especie una descripción morfológica detallada y observaciones, una ilustración, un mapa de distribución y una discusión de la ecología de la especie. Asimismo, se incluye una clave de identificación de las especies de Mimosa sección Habbasia de la Argentina.
\end{abstract}

Palabras clave: Flora argentina, Flora chaqueña, Mimosa sección Habbasia, serie Rojasianae.

Summary: Mimosa chacoënsis (Leguminosae), new record for the flora of Argentina. Mimosa chacoënsis, belonging to the section Habbasia series Rojasianae of genus Mimosa, is cited for the first time to Argentina, in the Formosa province. This species, endemic of the Dry Chaco ecoregion, has been previously registered only from Bolivia and Paraguay. As a result of this work the number of known species of Mimosa from the Argentine Flora increases to 56. A detailed morphological description and observations, an illustration, a distribution map, and a discussion of the ecology of this species are presented. Likewise, an identification key to species of Mimosa section Habbasia in Argentina is included.

Key words: Argentine Flora, Chacoan Flora, Mimosa section Habbasia, series Rojasianae.

\section{INTRODUCCIÓN}

El género Mimosa L. es uno de los más diversos de la familia Leguminosae, comprendiendo actualmente más de 530 especies (Barneby, 1991; Bessega \& Fortunato, 2011; Simon et al., 2011). Posee dos centros de diversificación importantes, uno en México y otro en Sudamérica austral, incluyendo el Planalto de Brasil y áreas adyacentes de Argentina, Bolivia y Paraguay (Barneby, 1991).

\footnotetext{
${ }^{1}$ Instituto de Recursos Biológicos, CIRN-CNIA, INTA, N. Repetto \& Los Reseros s.n., 1686 Hurlingham, Buenos Aires.

2 Consejo Nacional de Investigaciones Científicas y Técnicas (CONICET).

3 Facultad de Agronomía y Ciencias Agroalimentarias, Universidad de Morón, Cabildo 134, Morón, Buenos Aires, Argentina.

*Autor corresponsal: mmorales0007@gmail.com, morales. matias@inta.gob.ar
}

En la Argentina, este género posee hasta el momento 55 especies reconocidas (Fortunato et al., 2008; Morales, 2015) concentradas especialmente en la región mesopotámica del noreste del país (provincias de Misiones, Corrientes y Entre Ríos). Además presenta dos centros de diversificación minoritarios (1) en la región chaqueña: este de Jujuy, Salta, Catamarca y La Rioja, la totalidad de Formosa, Chaco y Santiago del Estero, y norte de Santa Fe, Córdoba y San Luis; y (2) en el área de las Yungas, el noroeste del país: centro de Jujuy, Salta, Tucumán y sudeste de Catamarca (Burkart, 1948).

De acuerdo con la variabilidad en indumento vegetativo, nectarios extraflorales y número de ciclos estaminales, Mimosa fue dividido en cinco secciones, de las cuales cuatro están representadas en Argentina: Batocaulon DC., Calothamnos Barneby, Habbasia DC. y Mimosa (Barneby, 1991). Esta clasificación infragenérica está siendo discutida a la luz de recientes estudios filogenéticos realizados sobre la base de genes del cloroplasto con 
optimización de caracteres morfológicos (Bessega \& Fortunato, 2011; Simon et al., 2011).

La sección Habbasia exhibe pocos representantes en la flora nativa argentina donde sólo cuatro de las más de 78 especies que presenta han sido citadas para este país: tres de la serie Habbasia (M. pigra L., M. strigillosa Torrey \& Gray y M. tweedieana Barneby ex Glazier \& Mackinder) y una de la serie Bipinnatae DC.: M. somnians Humb. \& Bonpl. ex Willd. (Barneby, 1991; Fortunato et al., 2008).

En el proceso de revisión de Mimosa para Argentina se ha registrado por primera vez para este país la presencia de $M$. chacoënsis Barneby \& Fortunato, especie perteneciente a la sección Habbasia serie Rojasianae Barneby, la cual es analizada.

\section{Materiales y Métodos}

En el contexto de la revisión del género Mimosa para la Flora Argentina, se analizaron especímenes de herbarios argentinos y extranjeros: BAB, BAF, CTES, FCQ, MO, NY y SI. Los mismos se identificaron y contrastaron con los tipos nomenclaturales y las propuestas taxonómicas vigentes (Barneby, 1991).

\section{Tratamiento taxonómico}

M. chacoënsis pertenece a la sección Habbasia serie Rojasianae por presentar flores tetrámeras, diplostémonas, con corola 1-nervada, hojas con estípulas plurinervadas, ausencia de espículas entre los pares de pinnas y los foliólulos con venación broquidódroma ramificándose hacia su mitad superior. Dentro de la serie Rojasianae, es fácilmente distinguible de sus vecinas, M. josephina Barneby (distribuida en Bolivia) y M. rojasii Hassl. (de Brasil y Paraguay), por la presencia de 11-14 pares de foliólulos por pinna, inflorescencias espiciformes y filamentos apenas unidos en su porción basal (Barneby, 1991).

Mimosa chacoënsis Barneby \& Fortunato. Brittonia 39: 169, fig. 3. 1987. Tipo: Paraguay. Presidente Hayes, Fortín Estancia Ayala (Nanawa), I-1981, Arenas s.n. (Holotipo BAF! [ex BACP 2386]; Isotipos CTES!, NY!). Fig. 1.

Subarbustos herbáceos, erectos, ascendentes o procumbentes, con ramas de hasta $1 \mathrm{~m}$ de largo. Tallos inermes, híspidos. Hojas con estípulas de 1,5-3,5 × 1-3 mm, 9-11-nervadas, pubérulas, cartáceas, persistentes; raquis primario de $50-80 \mathrm{~mm}$ de largo; pecíolo de 6-23 × 0,5-1 mm; pinnas 7-9 pares, sin espícula, raquis secundario de $15-20 \mathrm{~mm}$ de largo; foliólulos 11-14 pares por raquis, de 4-6 × 1-2 mm, oblongos, obtusos, 4-5-nervados, con venación secundaria broquidódroma que se ramifica en la mitad superior del foliólulo, glabros en el haz y pubescentes en el envés. Inflorescencias espiciformes, con pedúnculos de 5-10,3 cm de largo; espigas de 15-70 × 4-5 mm; brácteas florales de $1 \mathrm{~mm}$ de largo, plurinervadas, caducas. Flores tetrámeras, diplostémonas; cáliz de 0,2-0,5 $\mathrm{mm}$ de largo incluyendo las cilias, campanulado, papiráceo-membranáceo, ciliadodenticulado, glabro; corola de 2-2,5 mm de largo, turbinada, 1-nervada, glabra, con los lóbulos pilosos; estambres con filamentos exsertos, de 5,5-6,5 mm de largo, soldados alrededor del ovario rosado-pálidos; ovario de 0,2 $\mathrm{mm}$ de largo, hirsuto. Craspedios de 20-42 × 4,5-6 mm, oblongos, acuminados, rectos o levemente ondulados, 1-7-seminados, uniformemente híspidos y villosos sobre valvas y replum. Semillas de 3-4 $\times 2-3 \mathrm{~mm}$, obovoides, comprimidas.

Distribución geográfica y ecología. M. chacoënsis es un endemismo de la ecorregión del Chaco Seco (delimitación según Olson et al., 2001), que se suponía restricto a la región chaqueña paraguayo-boliviana (Barneby \& Fortunato, 1987; Barneby, 1991; Neill, et al., 2014; Atahuachi et al., 2016). El presente trabajo confirma su presencia en Argentina (Fig. 2), también en el área del Chaco Seco. De acuerdo con las observaciones de campo, crece generalmente en suelos arenosos, sobre dunas, pastizales, lugares alterados y bosques xéricos.

Material selecto examinado. ARGENTINA. Prov. Formosa: Dpto. Patiño, Muchutti, $10 \mathrm{~km}$ Norte de Las Lomitas, 4-XII-1978, Bordón s.n. (CTES 5719). BOLIVIA. Dpto. Chuquisaca: Prov. Carlos Calvo, El Salvador-Cimboc, Laguna El Panta, 21I-1992, Saravia Toledo \& Nelson 10312 (BAB); El Salvador, 8-XII-1992, Pensiero \& Marino 4379 (NY, SI). Dpto. Santa Cruz: Prov. Cordillera, Estancia Perforación y alrededores, XI-2001, Fuentes 3384 (MO). PARAGUAY. Dpto. Alto Paraguay: a $200 \mathrm{~m}$ de la delegación de Nueva Asunción, 18-XI-1992, Degen 2899 (FCQ, MO); Palmar de las Islas, 12-III1989, Mereles 2849 (FCQ); Nueva Asunción, 60 km 
M. Morales y R. H. Fortunato - Mimosa chacoënsis, nueva cita para Argentina

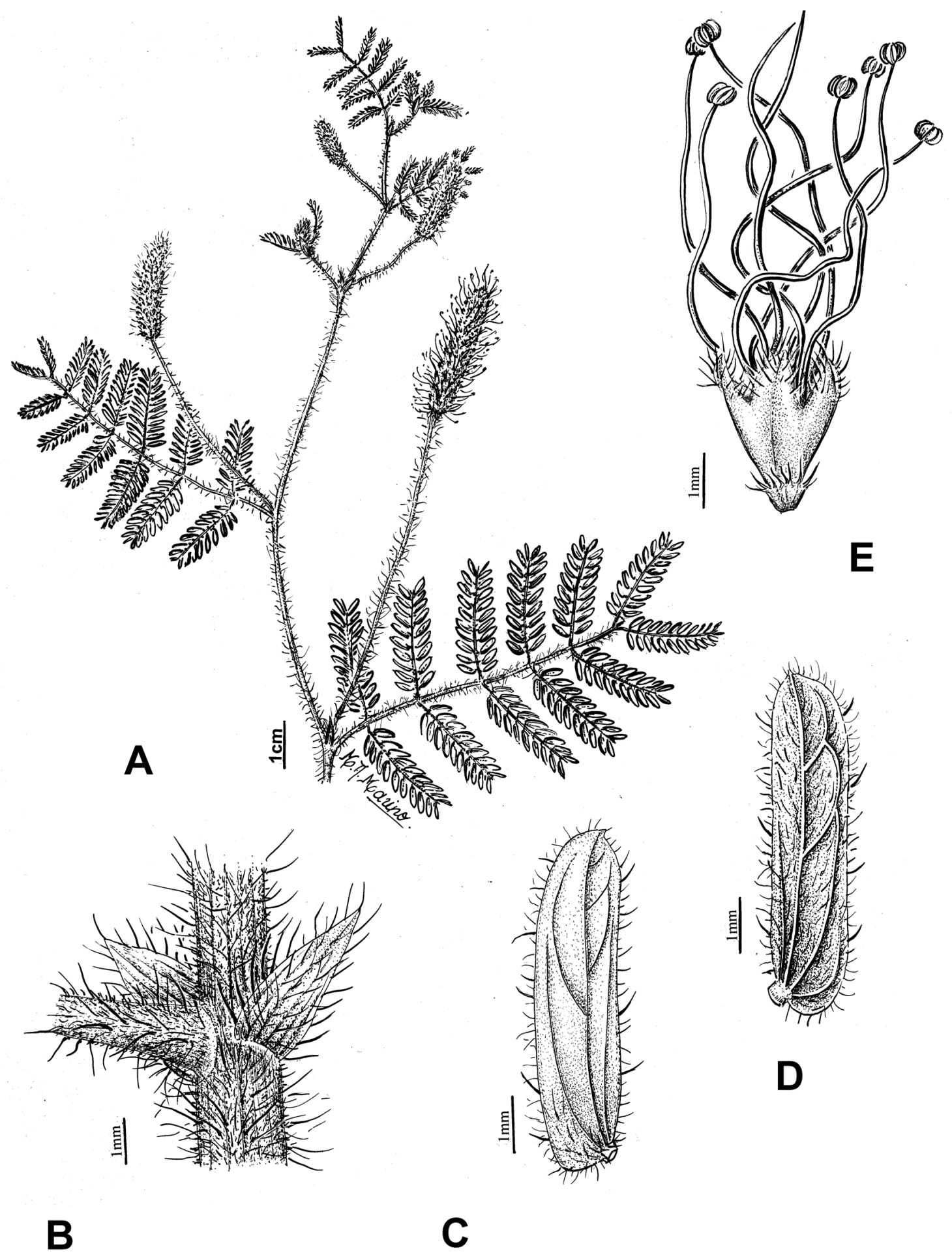

Fig. 1. Mimosa chacoënsis. A: Rama florífera. B: Detalle de nudo caulinar y estípulas. C: Foliólulo, haz. D: Foliólulo, envés. E: Flor. Dibujado por A. Marino (Bordón s.n., CTES 5719). 


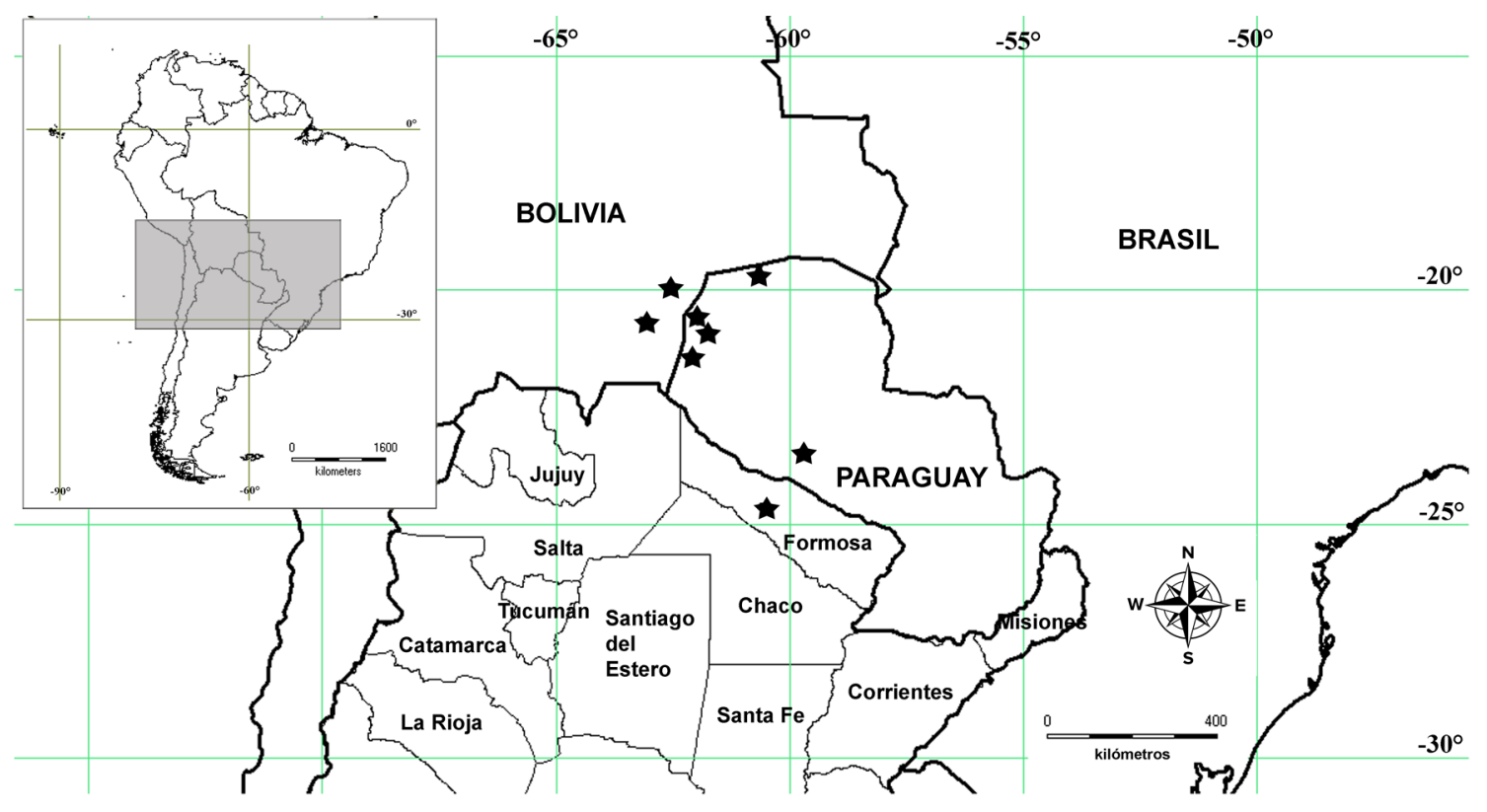

Fig. 2. Distribución geográfica de Mimosa chacoënsis.

al Oeste de Estancia La Patria, 7-XII-1992, Nicora et al. 9753 (BAB); ruta Transchaco, $25 \mathrm{~km}$ Sur de Nueva Asunción, 12-XII-1987, Schinini \& Palacios 25664 (BAB, FCQ, MO). Dpto. Boquerón: Between Parque Nacional Teniente Agripino Enciso y Nueva Asunción, 27-I-1995, Zardini \& Acosta 42248 (BAB); proposed National Park Médanos del Chaco, 13-XII1998, Zardini \& Duarte 49715 (BAB); 12-XII-1998, Zardini \& Duarte 49672, 49596 (BAB).

El espécimen argentino se ajusta a la descripción original (Barneby \& Fortunato, 1987) y la circunscripción propuesta por Barneby (1991) (Fig. 1). De acuerdo a los estudios en ejecución, la serie
Habbasia en Argentina estaría representada por cinco especies: M. chacoënsis, M. pigra, M. somnians, $M$. strigillosa y $M$. tweedieana, las que se diferencian por caracteres morfológicos vegetativos y florales.

Gracias a esta nueva cita, el presente trabajo también permite confirmar a la serie Rojasianae en Argentina, previamente exclusiva de Bolivia y Paraguay (Barneby, 1991). De esta forma, hasta el momento se registran tres series de la sección Habbasia en el país: serie Bipinnatae (con $M$. somnians Humb. \& Bonpl. ex Willd.), serie Habbasia (con M. pigra y M. tweedieana) y serie Rojasianae (con M. chacoënsis).

\section{Clave para las especies argentinas de Mimosa sección Habbasia}

1. Corola 3-11-nervada. M. somnians

1a. Corola 1-nervada. 2

2. Subarbusto cespitoso, decumbente, con tallos radicantes. M. strigillosa

2a. Subarbusto sin tallos radicantes. 3

3. Tallos inermes. Inflorescencias espiciformes. M. chacoënsis

3a. Tallos aculeados. Inflorescencias en capítulos globosos o subglobosos. 4

4. Estípulas densamente estrigoso-híspidas, con venación muy poço visible, 1-5-nervadas. M. pigra

4a. Estípulas generalmente glabras o estrigosas, con venación visible, 6-15-nervadas. M. tweedieana 


\section{Agradecimientos}

Agradecemos a los curadores de los herbarios visitados por el acceso a los materiales depositados en los mismos y los préstamos otorgados para el estudio del género, así como a Angélica Marino por la ilustración de la especie.

\section{Bibliografía}

ATAHUACHI, M., M. L. VAN DER BENT, J. R. I. WOOD, G. P. LEWIS \& C. E. HUGHES. 2016. Bolivian Mimosa (Leguminosae, Mimosoideae): three new species and a species checklist. Phytotaxa 260: 201-222.

BARNEBY, R. C. 1991. Sensitivae Censitae:Adescription of the genus Mimosa Linnaeus (Mimosaceae) in the New World. Mem. New York Bot. Gard. 65: 1-835.

BARNEBY, R. C. \& R. H. FORTUNATO. 1987. Four diplostemonous species of Mimosa (Mimosaceae) from Paraguay and eastern Bolivia. Brittonia 39: 165-174.

BESSEGA, C. \& R. H. FORTUNATO. 2011. Section Mimadenia: its phylogenetic relationships within the genus Mimosa (Leguminosae, Mimosoideae) using plastid trnL-F sequence data. Austr. Syst. Bot. 24: 104-110.

FORTUNATO, R. H., R. BEYHAUT, R. L. COSTA BORTOLUZZI, E. V. GÓMEZ SOSA, P. IZAGUIRRE, H. C. LIMA, S. T. S. MIOTTO, L. R. MOURA BAPTISTA \& E. A. ULIBARRI. 2008. Fabaceae. En: ZULOAGA, F., O. MORRONE \& M. J. BELGRANO (eds.), Catálogo de Plantas Vasculares del Cono Sur 2: 2078-2251. Actualizado en: ZULOAGA, F. O. \& M. J. BELGRANO (eds.). 2015. Catálogo de las plantas vasculares del Cono Sur (Argentina, sur de Brasil, Chile, Paraguay y Uruguay). Disponible en: $<$ http://www.darwin.edu. ar/Proyectos/FloraArgentina/fa.htm $>$ [consultado el 16 de mayo de 2017].

MORALES, M. 2015. Presencia de Mimosa regnellii (Mimosoideae, Leguminosae) en Argentina. Bol. Soc. Argent. Bot. 50: 413-418.

NEILL, D. A.; ATAHUACHI, M.; RICO, L.; KLITGAARD, G.; LEWIS, G.; BARHAM, J.; VARGAS, E. 2014. Fabaceae. En: Jørgensen, P. M., M. H. Nee \& S. Beck (eds.), Catálogo de las Plantas Vasculares de Bolivia, pp. 611-704. Missouri Botanical Garden Press, Saint Louis.

OLSON, D. M., E. DINERSTEIN, E. D. WIKRAMANAKAYE, N. D. BURGESS, G. V. N. POWELL, E. C. UNDERWOOD, J. A. D'AMICO, I. ITOUA, H. E. STRAND, J. C. MORRISON, C. J. LOUCKS, T. F. ALLNUTT, T. H. RICKETTS, Y. KURA, J. F. LAMOREUX, W. W. WETTENGEL, P. HEDAO \& K. R. KASSEM. 2001. Terrestrial ecoregions of the World: a new map of life on earth. Bioscience 51: 933-938.

SIMON, M. F., R. GRETHER, L. P. DE QUEIROZ, T. E. SäRKINEN, V. F. DUTRA \& C. E. HUGHES. 2011. The evolutionary history of Mimosa (Leguminosae): toward a phylogeny of the sensitive plants. Am. $J$. Bot. 98: 1201-1221.

Recibido el 30 de mayo de 2017, aceptado el 26 de octubre de 2017. Editor: Diego Gutierrez. 
